\title{
A New Component in the Radio Continua of PNe
}

\author{
S. Casassus* ${ }^{*}$ L.-Å. Nyman ${ }^{\dagger, * *}$, T.J. Pearson ${ }^{\ddagger}$ and A.C. Readhead ${ }^{\ddagger}$ \\ *Departamento de Astronomía, Universidad de Chile, Santiago, Casilla 36-D, Chile \\ ${ }_{\dagger}^{\dagger}$ European Southern Observatory, Alonso de Cordova 3107, Casilla 19001, Santiago 19, Chile \\ ** Onsala Space Observatory, 43992 Onsala, Sweden \\ ¥Owens Valley Radio Observatory, California Institute of Technology, Pasadena, CA 91125
}

\begin{abstract}
A byproduct of experiments designed to map the CMB is the recent detection of a new component of foreground galactic emission. The anomalous foreground at $10-30 \mathrm{GHz}$, unexplained by traditional emission mechanisms, correlates with 100 mum dust emission, and is thus presumably due to dust.

Is the anomalous foreground ubiquitous in the Galaxy? I will present evidence obtained with the CBI and SIMBA+SEST supporting the existence of the new component in the ISM at large, and in specific objects, in the form of a $31 \mathrm{GHz}$ excess over free-free emission in PNe.
\end{abstract}

Keywords: planetary nebulae: individual (NGC 7293, NGC 7009, NGC 6369), radio continuum: ISM, radiation mechanisms: general, infrared: ISM, ISM: dust

PACS: $98.38 . \mathrm{Ly}$; 98.58.Li; 98.38.-j;

\section{INTRODUCTION}

The RING5M experiment (Leitch et al. 1997) discovered an anomalous component of Galactic radio emission in the direction of translucent clouds about the North celestial pole. The $14-32 \mathrm{GHz}$ and $100 \mu \mathrm{m}$ intensities correlate, as expected from dust clouds photoionised by the diffuse UV field. But the corresponding $\mathrm{H} \alpha$ emission is absent. An explanation in terms of a hot plasma at $\sim 10^{6} \mathrm{~K}$, heated by shocks, is discarded by Draine \& Lazarian (1998a) on energetic grounds.

The anomalous emission, also called 'foreground X', is also present in the COBE data (Kogut et al. 1996) as a DMR-DIRBE correlation signal. A band-by-band linear model between the 31.5, 53, $90 \mathrm{GHz}$ DMR bands and the $100 \mu \mathrm{m}, 140 \mu \mathrm{m}$ and $240 \mu \mathrm{m}$ DIRBE bands gives a tight correlation slope at $31.5 \mathrm{GHz}(>6 \sigma)$, but loose at $90 \mathrm{GHz}$. The DMR-DIRBE signal has a flat spectrum, with $\alpha \sim+0.3 \pm 0.4$ (with $I_{v} \propto v^{\alpha}$ in Jy), and is tentatively interpreted as a mixture of traditional dust and free-free emission by Kogut et al. (1996).

The radio-IR correlations in the diffuse ISM are confirmed by de Oliveira-Costa et al. (1999); Finkbeiner et al. (1999); de Oliveira-Costa et al. (2002); Lagache (2003); Finkbeiner et al. (2002); Finkbeiner (2004). In particular Finkbeiner et al. (1999) report that the radioIR signal is not the Rayleigh-Jeans tail of the ISM dust: the DMR-DIRBE signal is at higher levels than predicted from the far-IR data, by factors of 1.2, 2.4 and 90 at 90 , 53 , and $31 \mathrm{GHz}$, respectively. de Oliveira-Costa et al. (2004) disprove the synchrotron interpretation by Bennett et al. (2003) of the radio-IR correlation as a WMAP foreground.
The idea that charged very small grains (VSGs) may rotate at frequencies reaching observable radio frequencies originated with Erickson (1957) and Hoyle \& Wickramasinghe (1970), and was revived in an accurate model by Draine \& Lazarian (1998b) to explain the RING5M experiment. The spectral energy distribution (SED) of spinning dust rises steeply with frequency $(\alpha \sim 3)$, and then drops-off with a Boltzmann factor. Draine \& Lazarian (1998b) obtain a peak at $25-30 \mathrm{GHz}$ and intensity levels slightly short of the anomalous foreground in the RING5M experiment.

The spinning dust emission mechanism may be understood in crude terms by assuming equipartition in the rotational degrees of freedom: $\frac{1}{2} I \omega^{2}=k T$. For a spherical and homogeneous grain, with $I=\frac{2}{5} m R^{2}$, a radius $R=N 10^{-10} \mathrm{~m}$, and a mass density $\rho=1 \mathrm{~kg} \mathrm{~m}^{-3}$, the rotation frequency is $v=210^{3} \sqrt{T / N^{5}} \mathrm{GHz}$. A typical dust temperature is $T \sim 25 \mathrm{~K}$, and a very small grain cannot be much less than 100 atoms in radius (so $N=10^{2}$ ), therefore $v \sim 30 \mathrm{GHz}$.

As explained by Draine \& Lazarian (1999) dust emission traditionally refers to thermal fluctuations in the charge distribution (or vibrational emission). Magnetic dipole emission arises from fluctuations in the grain magnetization. Draine \& Lazarian (1999) show that the inclusion of the grain response to the magnetic field enhances the grain emissivities at radio-frequencies by adding a term to the grain cross-section, $j_{v}=n_{\text {grains }}\left(C_{e}(v)+\right.$ $\left.C_{m}(v)\right) B_{v}(T)$, to an extent that rivals spinning-dust for the case of ferromagnetic grains. Unlike spinning dust, magnetic dipole emission at radio-frequencies stems from the large grains. 
We report on observations obtained with the CBI supporting the existence of the new component in specific targets. The CBI is a radio-interferometer operating in 10 channels over $26-36 \mathrm{GHz}$. Its 45 arcmin primary beam and 4 arcmin resolution is well suited to map extended sources, such as the Helix nebula and the dark-cloud LDN 1622. The CBI also allows measuring the $31 \mathrm{GHz}$ flux densities from compact and isolated objects, such as young planetary nebulae ( $\mathrm{PNe}$ ), which we complement with SIMBA $250 \mathrm{GHz}$ data.

\section{A RADIO-BRIGHT DARK CLOUD: LDN 1622}

LDN 1622 is the first specific target where the anomalous emission was detected. Finkbeiner et al. (2002) reported the marginal detection of a positive $5-10 \mathrm{GHz}$ index, and Finkbeiner (2004) detected a negative index above $30 \mathrm{GHz}$ using the WMAP data.

The CBI has finer spatial resolution than WMAP, which allows us to use morphological information to link the $31 \mathrm{GHz}$ emission with the vSGs. Fig. 1 shows an overlay of a super-resolved CBI image on the four IRAS bands. The images are MEM models taylored to the CBI, and the CBI $u v$-plane has been simulated on the IRAS templates.

Inspection of Fig. 1 shows that the CBI image resembles most the IRAS $12 \mu \mathrm{m}$ and $25 \mu \mathrm{m}$ band images, and not the $100 \mu \mathrm{m}$ one. VSGs dominate the shorter-wavelength IRAS bands, and contribute little to the $100 \mu \mathrm{m}$ band. We conclude that the $31 \mathrm{GHz}$ emission is linked to the VSG population, as expected from spinning-dust.

\section{THE HELIX NEBULA}

The CBI observations of the Helix PN show an excess at $31 \mathrm{GHz}$ at about $3 \sigma$, which cannot be explained by free-free emission. In Fig. 2, taken from Casassus et al. (2004), we set up a SED for the Helix, including upper limits at $250 \mathrm{GHz}$ obtained with SIMBA on SEST. The SED shows a low frequency rise that requires excesive emission measure for optically thick free-free emission.

The Helix is renowned for its cometary knot complex. With a turn-over frequency of $v_{T} \sim 1 \mathrm{GHz}$, the knots would be optically thin and stand out at $31 \mathrm{GHz}$, and yet be optically thick and faint at $0.408 \mathrm{GHz}$. But the required filling-factor is $\varepsilon \sim 1 / 1000$, while comparing the radio and $\mathrm{H} \beta$ fluxes gives $\varepsilon \sim 1$.

The morphology of the $31 \mathrm{GHz}$ emission also supports an origin other than free-free emission. In Fig. 3 the CBI image resembles a mixture of the far-IR emission and the
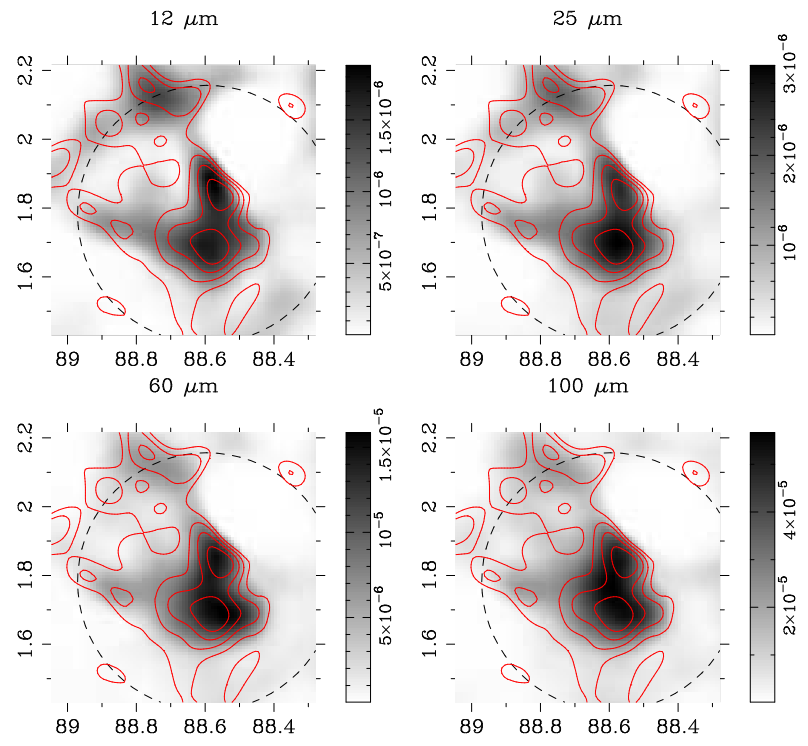

FIGURE 1. Intensity units are $\mathrm{MJy} \mathrm{sr}^{-1}$, and the contours follow the CBI $31 \mathrm{GHz}$ MEM model.

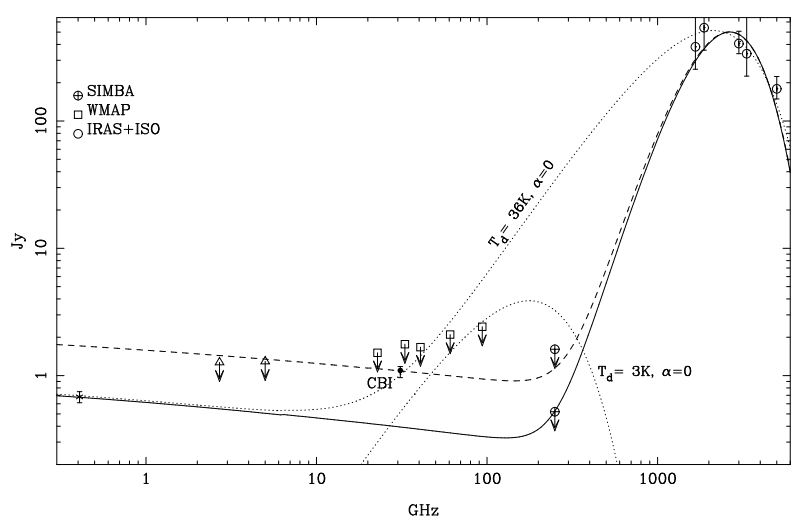

FIGURE 2. SED of the Helix PN. The solid line is a theoretical SED designed to fit the measurement at $408 \mathrm{MHz}$ and the IRAS and ISOPHOT data. The dashed line is a model SED designed to fit the CBI points, drawn to emphasize the disagreement with low frequency observations. The two SIMBA points correspond to two levels of rigor in the data reduction. The black-body curves in dotted lines rule-out cold grains as responsible for the $31 \mathrm{GHz}$ excess.

$\mathrm{H} \beta$ maps. To obtain comparable maps we simulate CBI observations on dust and free-free emission templates (see Casassus et al. 2004). H $\beta$ should be proportional to free-free emission if the electronic temperature $T_{e}$ is constant, and if extinction is negligible at $\mathrm{H} \beta$ (the Helix has negligible Balmer extinction, O’Dell 1998; Henry et al. 1999).

$T_{e}$ variations cannot explain the anomalous emission map in Fig. 3. For a free-free continuum at $31 \mathrm{GHz}$, we can produce the $T_{e}$ map shown on Fig. 4 by dividing the 
$31 \mathrm{GHz}$

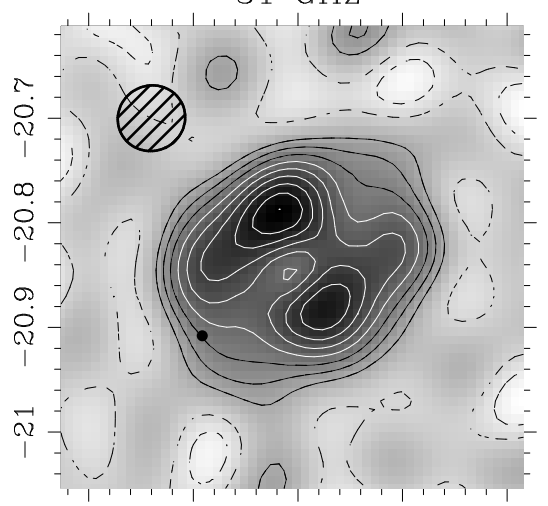

$60 \mu \mathrm{m}$

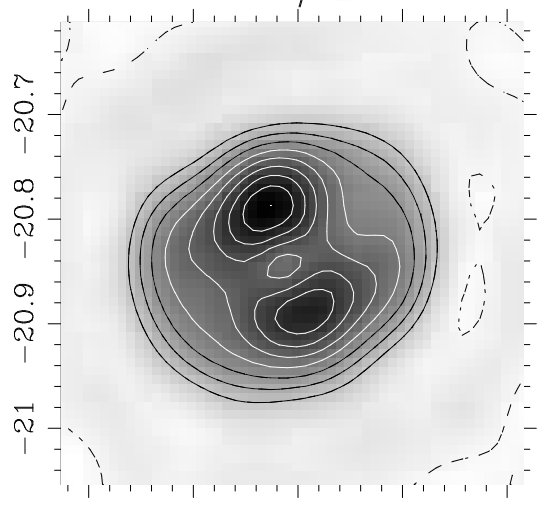

$180 \mu \mathrm{m}$

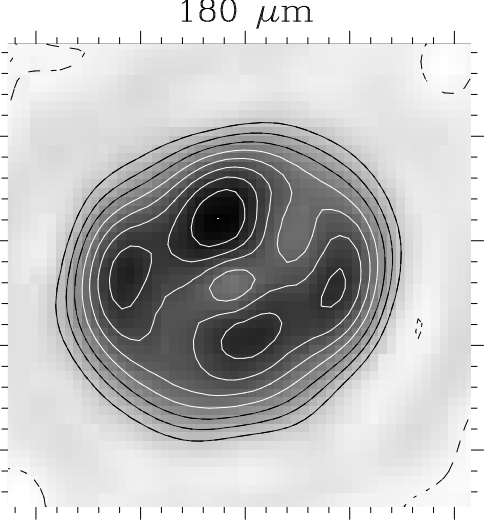

$\mathrm{H} \beta$

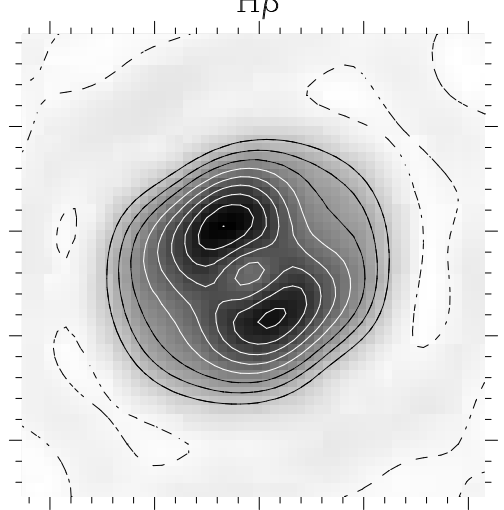

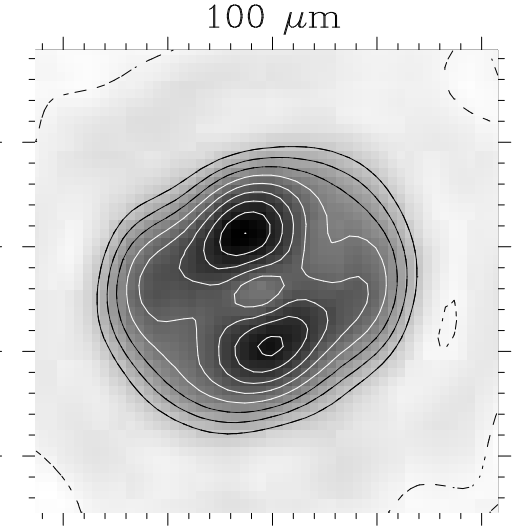

$31 \mathrm{GHz}-\mathrm{a}_{\mathrm{H} \beta}^{\mathrm{II}} \mathrm{H} \beta$

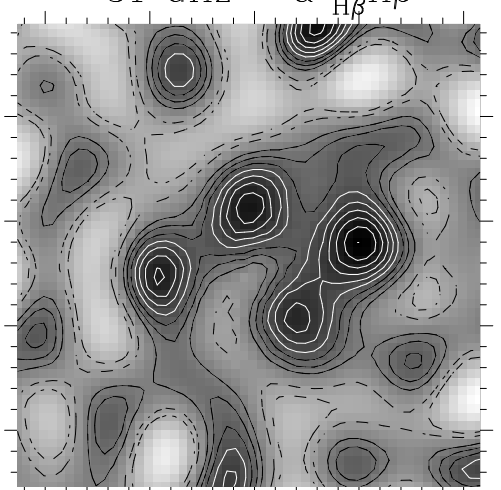

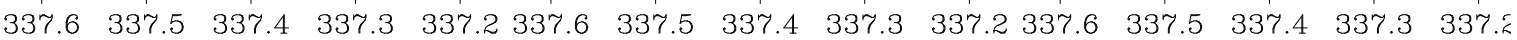

FIGURE 3. The Helix at $31 \mathrm{GHz}, 100 \mu \mathrm{m}, 180 \mu \mathrm{m}, 60 \mu \mathrm{m}$, and $\mathrm{H} \beta$, as seen by the CBI: CBI observations have been simulated on the templates for a faithful comparison. The image on the lower right results after subtracting the appropriately scaled $\mathrm{H} \beta$ template from the CBI image (see Casassus et al. (2004)), and shows the anomalous emission. The beam ellipse shown on the $31 \mathrm{GHz}$ image is the same for all images.

$31 \mathrm{GHz}$ and $\mathrm{H} \beta$ images. The $T_{e}$ variations do not follow the anomalous emission map. $T_{e}$ peaks on the nebular ring, while the photoionisation models in Henry et al. (1999) and the arguments given by O'Dell (1998) point at a radially decreasing $T_{e}$.

\section{GHZ EXCESS IN COMPACT PNE}

We set up the SEDs of a sample of compact PNe using the CBI and SIMBA flux densities. A subset of the sample shows a drop at $250 \mathrm{GHz}$ compared to the free-free continuum that can be extrapolated from the CBI and literature data. We give two examples in Fig. 5 and Fig. 6. The $250 \mathrm{GHz}$ fluxes fall well below the expected level of free-free emission in the PNe NGC 3242 (9 $\sigma$ deviation), NGC $6369(7 \sigma)$, NGC $3918(6 \sigma)$, NGC $7009(5.5 \sigma)$, $\operatorname{NGC} 6572(3.5 \sigma)$.

The SIMBA+SEST data points at $250 \mathrm{GHz}$ were obtained in 3 different observing runs in the case of NGC 7009, and two for NGC 6369. The SIMBA bolome-

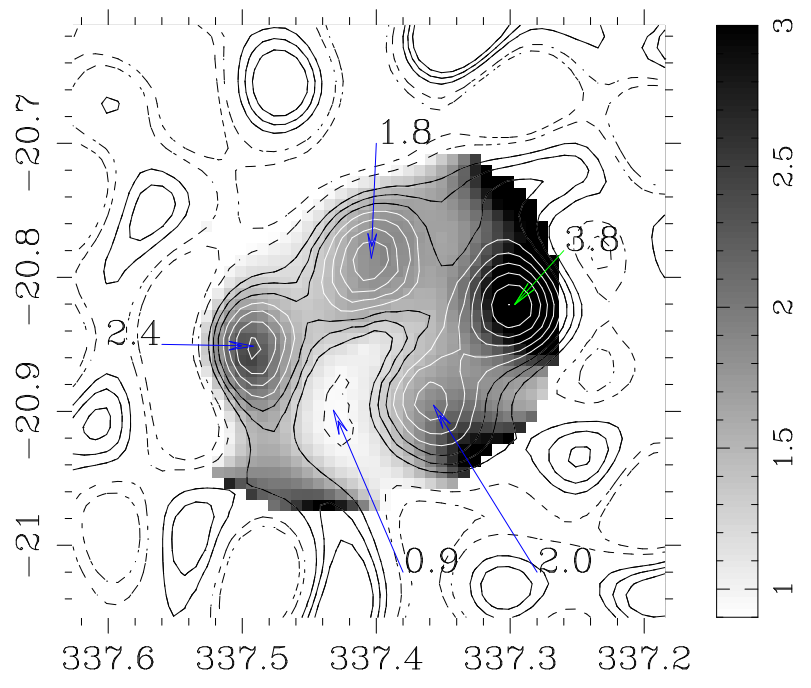

FIGURE 4. $T_{e}$ map in gray scale, with an overlay of the anomalous $31 \mathrm{GHz}$ emission from Fig. 3 in contours. Units are $10^{4} \mathrm{~K}$. 


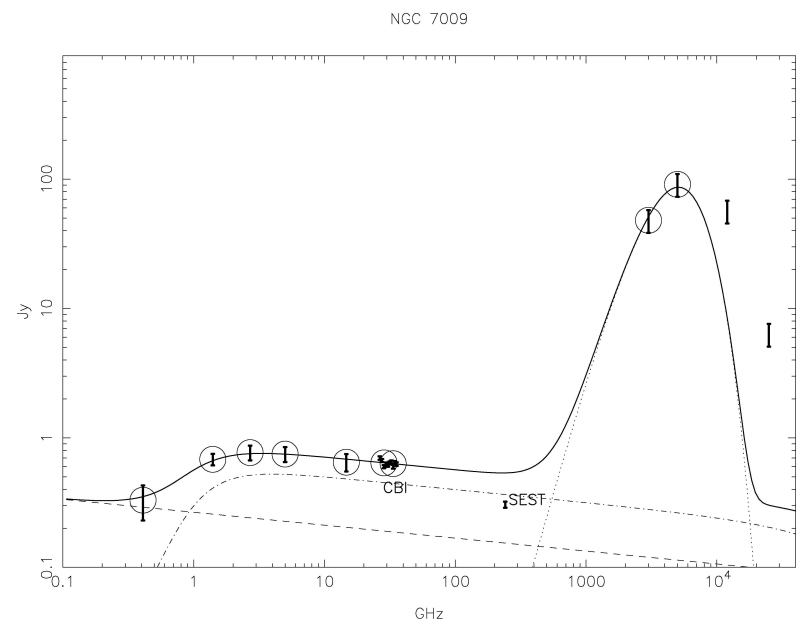

FIGURE 5. SEDs of the compact PN NGC 7009. The dotdashed and dashed lines correspond to a core-halo free-free fit to the circled data.

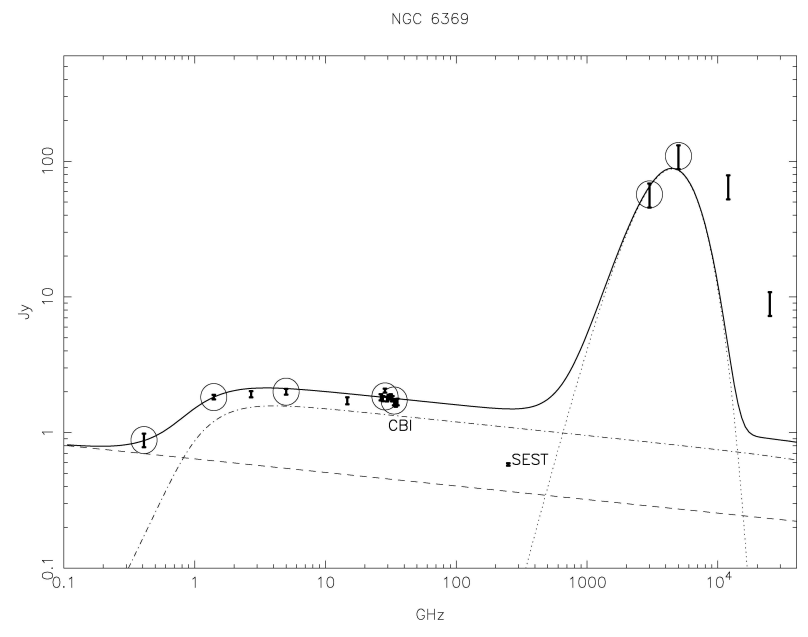

FIGURE 6. SEDS of the compact PN NGC 6369.

ter maps confirm previous heterodyne data. Additionally, we corroborate our $250 \mathrm{GHz}$ flux measurements by comparison with Hoare et al. (1992) within $20 \%$ uncertainties, for the three objects we have in common (NGC 6572, NGC 6302, and NGC 6537).

An interpretation of the drop-off above $30 \mathrm{GHz}$ in terms of extinction due to $\mathrm{cm}$ or $\mathrm{mm}$-sized grains implies absurd dust-to-gas mass ratios (of order 1). Fitting the NGC 6369 SED with a synchrotron component requires that all of the 5-30 GHz emission be synchrotron, modulated by an absurdly cold free-free screen at $\sim 100 \mathrm{~K}$.

\section{CONCLUSION}

The Helix nebula provides evidence for a new emission mechanism at $31 \mathrm{GHz}$, other than synchrotron or freefree emission. Since VSGs are not expected to survive the PN phase, and since the dust in the Helix is relatively hot, this result supports magnetic dipole emission as a candidate mechanism for the $31 \mathrm{GHz}$ excess.

We obtained a $30 \mathrm{GHz}$ image of the dark cloud LDN 1622, which is indicative of spinning dust emission.

The compact PNe provide the strongest evidence for the existence of the new component in the context of photoionised nebulae. Free-free represents $50 \%$ of the $30 \mathrm{GHz}$ flux in the extreme case of NGC 6369.

\section{ACKNOWLEDGMENTS}

S.C acknowledges support from Fondecyt grant 1030805, and from the Chilean Center for Astrophysics FONDAP 15010003.

\section{REFERENCES}

Bennett, C.L., Hill, R.S., Hinshaw, G., et al. 2003, ApJS, 148, 97

Casassus, S., Readhead, A.C.S., Pearson, T.J., et al. 2004, ApJ, 603, 599

de Oliveira-Costa, A., Tegmark, M., Gutiérrez, C.M., et al. 1999, ApJ, 527, L9

de Oliveira-Costa, A, Tegmark, M., Finkbeiner, D.P., et al. 2002, ApJ, 567, 363

De Oliveira-Costa, A., Tegmark, M., Davies, R.D., et al. 2004, ApJ, 606, L89

Erickson, W.C. 1957, ApJ, 126, 480

Draine, B.T., \& Lazarian, A. 1998a, ApJ, 494, L19

Draine, B.T., \& Lazarian, A. 1998b, ApJ, 508, 157

Draine, B.T., \& Lazarian, A. 1999, ApJ, 512, 740

Finkbeiner, D.P. 2004, ApJ, 614, 186

Finkbeiner, D.P., Davis, M., Schlegel, D.J. 1999, ApJ, 524, 867

Finkbeiner, D.P., Schlegel, D.J., Frank, C., Heiles, C. 2002, ApJ, 566, 898

Henry, R.B.C., Kwitter, K.B., Dufour, R.J. 1999, ApJ, 517, 782

Hoare, M.G., Roche, P.F., Clegg, R.E.S. 1992, MNRAS, 258, 257

Hoyle, F., \& Wickramasinghe, N.C. 1970, Nature, 227, 473

Kogut, A., Banday, A.J., Bennett, et al. 1996, ApJ, 460, 1

Lagache, G. 2003, A\&A, 405, 813

Leitch, E.M., Readhead, A.C.S., Pearson, T.J., Myers, S.T. 1997, ApJ, 486, L23

O’Dell, C.B. 1998, AJ, 116, 1346 